\title{
Detection of central veins using susceptibility weighted imaging (SWI) for discrimination between lesions in multiple sclerosis (MS) and cerebral small vessel disease (CSVD)
}

\author{
Rania Adel Anan ${ }^{1 *}$, Mohamed Ali El-Adalany², Tamer Mohamed Ibrahim Belal ${ }^{3}$ and Sabry Alam El-Dein Elmogy ${ }^{2}$
}

\begin{abstract}
Background: Conventional brain MRI cannot always distinguish between different white matter lesions. Susceptibility weighted imaging (SWI) can detect a sign called central vein sign which is suggested to discriminate between multiple sclerosis and other white matter lesions thus increasing the accuracy of MRI in MS diagnosis. This study aimed to evaluate the role of MR susceptibility weighted imaging in differentiating MS from CSVD lesions.

Results: The central vein sign (CVS) was found to have a premium diagnostic performance in diagnosis of MS with sensitivity $83.3 \%$, specificity $70 \%$, positive predictive value $80.6 \%$, and negative predictive value $73.7 \%$. When CVS positive lesions load was $\geq 44.24 \%$, the specificity of CVS in diagnosing MS raised to $100 \%$.
\end{abstract}

Conclusion: Detection of the CVS on SWI can reliably discriminate between MS and CSVD.

Keywords: Central vein sign, Multiple sclerosis, Cerebral small vessel disease, Susceptibility weighted imaging

\section{Background}

Multiple sclerosis (MS) is defined as an inflammatory autoimmune neurodegenerative disease of the central nervous system, characterized by inflammation, demyelination, gliosis, and neuro-axonal loss in lesions [1].

MRI is a common investigation which can support a clinical diagnosis of MS by detecting lesions in the brain and spinal cord [2]. It can also be used for monitoring the disease activity which helps to guide treatment decision and to decrease relapse occurrence and disease progression [3, 4].

With advancing neuro-imaging, more atypical cases were experienced and because white matter lesions are

\footnotetext{
* Correspondence: ronyanan87@gmail.com

'Specialist of Diagnostic and Interventional Radiology, Ministry of Health, Cairo, Egypt

Full list of author information is available at the end of the article
}

depicted in many CNS diseases, specific diagnostic tools are needed [5].

Multiple MRI protocols were evolved to detect MS plaques and differentiating them from other similar lesions. Regarding the pathogenesis of MS lesions, they were found to be typically perivenular, so special MR sequences were used to detect the veins within the MS plaques [6], this feature is called the "central vein sign," that would increase the accuracy of MRI in MS diagnosis [7].

Susceptibility-weighted imaging is one of the new MR sequences which can draw a venous map of the brain and can show the central vein in MS lesions [5].

Several recent studies using SWI in imaging of MS patients have revealed that SWI is more sensitive than conventional MR sequences (such as T2-weighted and FLAIR images) in detection of MS plaques and can 
Table $1 \mathrm{MRI}$ parameters used

\begin{tabular}{llll}
\hline Parameter & SWI & FIAIR & T2 \\
\hline Field of view (mm) & 230 & 230 & 230 \\
Matrix & $512 \times 256$ & 256 & 256 \\
Slice thickness (mm) & 3.6 & 5 & 5 \\
Voxel size & 0.9 & 0.9 & 0.9 \\
Repetition time (ms) & 77.3 & 6500 & 6735 \\
Echo time (ms) & 49.2 & 118.9 & 106 \\
Inversion time (ms) & - & 1967 & - \\
Acquisition time (ms) & 4 min & 4 min 18 s & 2 min 11 s \\
Flip angle & 15 & 90 & 160 \\
\hline
\end{tabular}

demonstrate many signal patterns not previously seen within the MS plaques [8].

This study aimed to evaluate the role of MR susceptibility-weighted imaging in differentiating MS from CSVD lesions.

\section{Methods}

Informed written consents were obtained from the patients' guardians according to the National Ethics Committee.

\section{Patients}

This prospective study included 50 patients who were classified into two groups: 30 patients (22 females and 8 males) were included in the first group, they were diagnosed clinically as MS, their ages ranged from 17 to 54 years. Patients with severe kidney disease or allergy to contrast medium, cardiovascular, cerebrovascular disease, or history of malignant disease were excluded. The second group included 20 patients (6 females and 14 males) with white matter lesions, possibly due to CSVD, their ages ranged from 36 to 70 years. Patients with prior brain stroke or history of malignant disease were excluded.

\section{MRI imaging}

All patients were examined by MRI $1.5 \mathrm{~T}$ system (GE SIGNA Explorer) with 16-channel head coil in supine position using a uniform protocol which includes axial T2-weighted and FLAIR sequences and SWI for detection of the CVS (Table 1).

If the referring physician suspects disease activity in MS patients, or if new lesion was detected, contrast enhanced T1-weighted sequence was done using gadolinium contrast medium with the dose $(0.3 \mathrm{mmol} / \mathrm{kg})$.

\section{Image analysis}

Two conjoint experienced neuroradiologists analyzed the data; they were totally blinded to patients' clinical history. They analyzed FLAIR and T2W images for detection and localization of hyperintense white matter lesions then they classify the lesions according to their location into periventricular or non-periventricular and the non-periventricular lesions were then sub-classified into juxta-cortical, deep white matter, and infratentorial. Only the lesions which were $3 \mathrm{~mm}$ or more in size were included and confluent lesions were excluded.

In patients with CSVD, only patients who had lesions classified as Fazekas 0-2 (according to Fazekas scale [9, 10]) were included. Fazekas 0 lesion was defined as single punctate lesion, Fazekas 1 lesions represented multiple punctate lesions. Fazekas 2 lesions included some lesions that were beginning confluence. In this group of patients, confluent lesions were also excluded.

SWI images were analyzed for depiction of a central vein in the white matter lesions. The MinIP SWI images were utilized to better identify the signal void of a vein.

The central vein sign was defined according to the standard radiological definition established in the North American Imaging in Multiple Sclerosis (NAIMS) guidelines $[11,12]$ which recommend that: (Fig. 1)

1) Lesions adopted a 'coffee bean' appearance with the vein appears as a thin hypointense central line

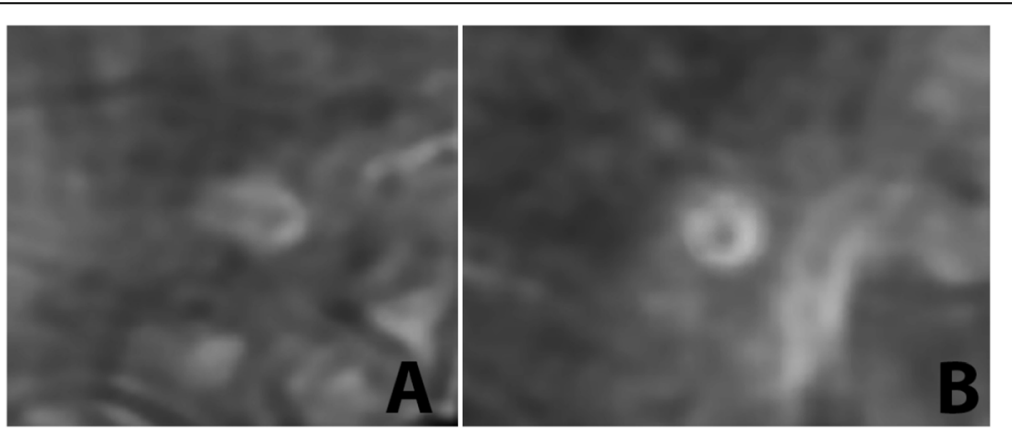

Fig. 1 Magnified SWI images showing the morphology of the central vein sign according to vein direction. a Lesion with "coffee bean" appearance in which a thin hypointense line (representing the CVS) is seen running through the lesion and intersecting it. b Lesion with "doughnut" appearance, in which a hypointense central circular dot is noted and representing the CVS 


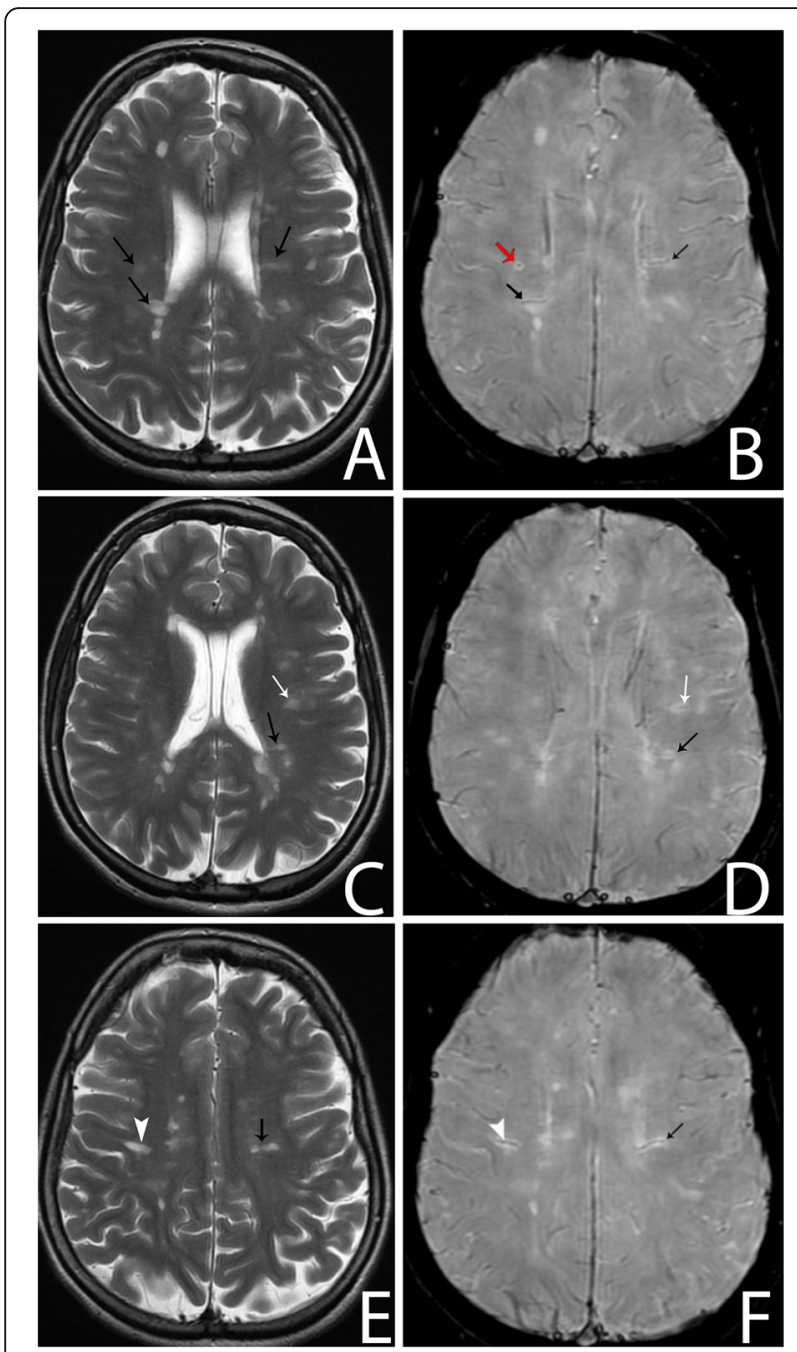

Fig. 2 Axial (a, c e) T2WI images show hyperintense MS periventricular (black arrows), deep white matter (arrowhead), and juxta-cortical lesions (white arrow). On corresponding (b, d f ) SWI images, a thin hypointense line representing the CVS is seen within the periventricular (black arrows), deep white matter (arrowhead), and juxta-cortical lesions (white arrow) while a central hypointense dot in the right periventricular lesion (red arrow) (when the MRI slice was parallel to the vein long axis) or a 'doughnut' appearance with the vein appears as a small hypointense central dot (when the MRI slice was perpendicular to the vein).

2) The vein runs partially or entirely through the lesion, but must be central in location regardless of the lesion's shape.

3) The vein is small in diameter $(<2 \mathrm{~mm})$.

When both evaluators were in agreement in detection of CVS, we defined the lesion as a CV positive lesion.

Exclusion criteria were also designed according to the standard radiological definition and included: lesion $<3$ $\mathrm{mm}$ in diameter; confluent lesion; lesion with multiple distinct veins and lesion that is poorly visible (due to motion or other MRI artifacts).

\section{Statistical analysis}

Descriptive statistics were calculated for the two groups of patients, including mean/median, percentage, and range (minimum-maximum values). Then, the ratios between number of CVS positive lesions and total number of lesions for each patient were calculated, in both groups. The same ratios were also calculated for lesions according to their distribution (periventricular, nonperiventricular). Mann-Whitney $U$ test was used to compare variables between both groups which include total number of lesions, periventricular, non-periventricular. SPSS software package version 22.0 was used to analyze the data. A value of $p<0.05$ was considered statistically significant.

\section{Results}

In this study, 50 patients were scanned: 30 of them were diagnosed clinically as MS, they included 22 females and 8 males with mean age $30.97 \pm 9.79$ years (range 17-54 years). The other 20 patients (including 6 females and 14 males with mean age $61.40 \pm 10.34$ years (range $36-$ 70 years)) were known to have ischemic white matter lesions related to cerebral small-vessel disease. The difference in age and sex between the two groups of patients was significantly different $(p<0.001)$.

Table $\mathbf{2}$ Lesion distribution presented as number and percentage

\begin{tabular}{|c|c|c|c|c|c|}
\hline & \multicolumn{4}{|c|}{ Total $(N=597)$} & \multirow[t]{3}{*}{ Test of significance } \\
\hline & \multicolumn{2}{|c|}{ MS } & \multicolumn{2}{|c|}{ CSVD } & \\
\hline & No & $\%$ & No & $\%$ & \\
\hline Total (periventricular and non-periventricular distributed) & 453 & 75.9 & 144 & 24.1 & $p<0.001^{*}$ \\
\hline Periventricular distributed & 181 & 39.9 & 43 & 29.9 & $p=0.02^{*}$ \\
\hline $\begin{array}{l}\text { Non-periventricular distributed: } \\
\text { a) Juxta-cortical } \\
\text { b) Deep white matter } \\
\text { c) Infratentorial }\end{array}$ & $\begin{array}{l}272 \\
93 \\
159 \\
20\end{array}$ & $\begin{array}{l}60 \\
34.1 \\
58.5 \\
7.4\end{array}$ & $\begin{array}{l}101 \\
30 \\
64 \\
7\end{array}$ & $\begin{array}{l}70.1 \\
29.7 \\
63.4 \\
6.9\end{array}$ & $\begin{array}{l}p<0.001^{*} \\
p=0.41 \\
p=0.39 \\
p=0.89\end{array}$ \\
\hline
\end{tabular}




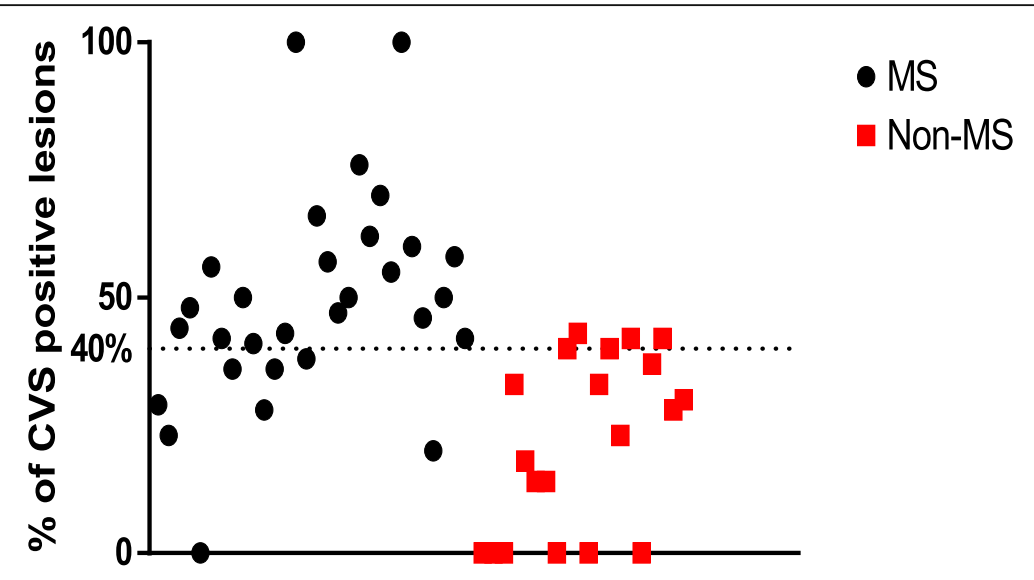

Fig. 3 Comparison of the proportion of peri-venous lesions seen in patients with MS versus patients with CSVD

Regarding disease duration in MS group, 83.3\% of MS patients suffered from the disease for $<5$ years and $16.7 \%$ of patients for $\geq 5$ years.

Based on the duration of MS disorder, the numbers of detected plaques for each patient were calculated in T2, FLAIR, and SWI sequences. It was found that the median numbers of detected plaques in patients with disease duration more than 5 years were $24(18-31)$ in T2W sequences, 25 (18-33) in FLAIR sequences, and 25 (14-31) in SWI sequences. However, the median numbers of plaques in patients with disease duration less than 5 years were $12(3-40), 13(3-37)$, and $12(3-38)$ in T2W, FLAIR, and SWI sequences, respectively. All MRI sequences used in this study showed significantly higher numbers of plaques in patients with disease duration more than 5 years than patients with disease duration less than 5 years $(p=0.01)$.

In MS patients, 453 lesions were detected, while 144 lesions were detected in patients with CSVD. Regarding lesion distribution, in MS patients, 181 (39.95\%) lesions were detected in the periventricular region, (Figs. 2 and 5) while $272(60 \%)$ lesions were detected in non-periventricular location and then classified as (93 (34.1\%) juxta-cortical, 159 (58.5\%) deep white matter, and 20 (7.4\%) infratentorial). In CSVD patients, $43(29.9 \%)$ lesions were detected in the periventricular region while $101(70.1 \%)$ lesions were detected in non-periventricular location and then classified as (30 (29.7\%) juxta-cortical, 64 (63.4\%) deep white matter, and 7 (6.9\%) cerebellar) (Table 2).

Table 3 Number and percentage of lesion with CVS in MS and CSVD patients

\begin{tabular}{llllll}
\hline & $\begin{array}{c}\text { MS lesions } \\
\text { (no }=453)\end{array}$ & CSVD lesions $(\mathrm{no}=144)$ & Test of significance \\
& No & $\%$ & No & $\%$ & \\
CVS positive & 223 & 49.22 & 37 & 25.69 & $p<0.001^{*}$ \\
CVS negative & 230 & 50.77 & 107 & 74.3 & \\
\hline
\end{tabular}

By analyzing the proportion of CVS-positive lesions in each patient, we found that the number of MS patients presented with CVS-positive lesions (29 patients with the proportion of CVS positive lesions in each patient ranging from 0 to $100 \%$, median $48 \%$ ) was significantly higher than CSVD patients (14 patients with the proportion of CVS positive lesions in each patient ranging from 0 to $44 \%$, median $25 \%$ ) (Fig. 3 ). This was statistically significant $(p=0.007)$.

A total of 223 out of 453 MS lesions (49.22\%) have demonstrated the CVS, most of them $(124 / 223,55.6 \%)$ seen in the periventricular region, while only 37 out of 144 CSVD lesions $(25.69 \%)$ were CVS-positive with $(15 / 37,40.5 \%)$ lesions had a periventricular distribution. This was statistically significant with $(p$ value $<0.001)$ (Table 3 ).

As regards the location of the CVS positive lesions, there was a statistically significant difference between the MS and CSVD patients in both periventricular and non-periventricular location (with the $p$ value $=0.004$ and 0.007 , respectively) as shown in Fig. 4. In MS patients, $68.5 \%(124 / 181)$ of periventricular lesions and $36.39 \%(99 / 272)$ of non-periventricular lesions were positive for CVS (Figs. 2 and 5), while in CSVD group, only $34.88 \%(15 / 43)$ of periventricular lesions and $21.78 \%(22 / 101)$ of non-periventricular were positive for CVS (Figs. 6 and 7).

The CVS was found to have a premium diagnostic performance in diagnosis of MS with the sensitivity $83.3 \%$, specificity $70 \%$, positive predictive value $80.6 \%$, and negative predictive value $73.7 \%$ (Table 4). When CVS positive lesions load was $44.24 \%$ and above, the specificity of CVS in diagnosing MS raised to $100 \%$ (Fig. 8).

\section{Discussion}

White matter lesions are usually depicted on brain MRI done for various indications. The majority of them caused by microangiopathy, but multiple sclerosis, is also considered an important cause. Conventional MRI 


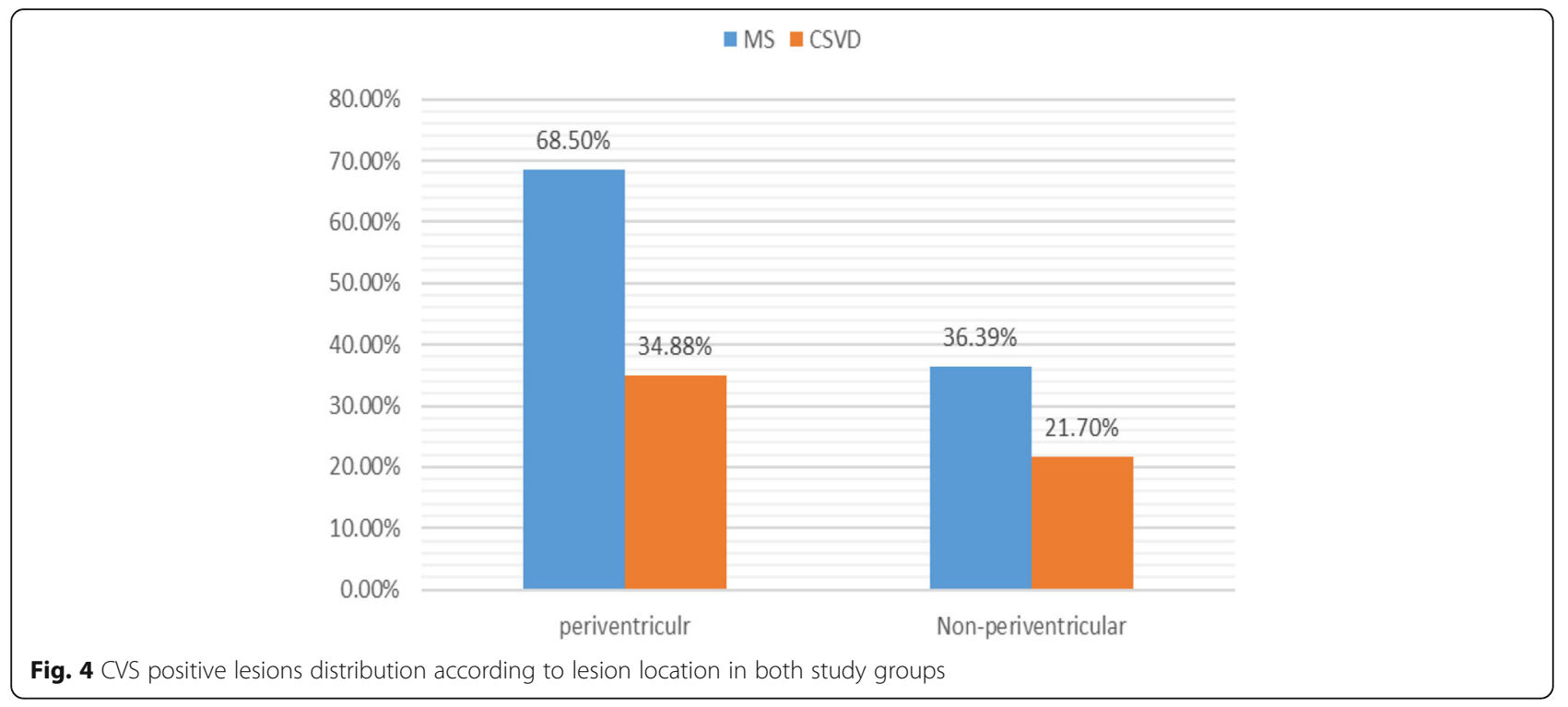

cannot accurately differentiate between these pathologies. SWI can detect the central vein sign which has been suggested to discriminate between MS and other white matter lesions as microangiopathic brain lesions [13].

This study revealed that central vein sign positive lesions were much more in MS (49.22\%) than in CSVD $(25.69 \%)$ with statistically significant difference $(p<$ 0.001). This is in agreement with a study done by Sparacia et al. [14], in which $40.9 \%$ of MS lesions versus $29.3 \%$ of CSVD lesions have demonstrated the central vein sign. This difference explains the different pathogenesis of both diseases.

It was also found that the proportion of CVS-positive lesions in patients with MS (median 48\%, range 0-100\%) was consistently much higher than in subjects with CSVD (mean 25\%, range 0-44\%). This copes with Tallantyre et al. study [15] which revealed higher proportion of perivenous lesions in patients with MS (mean $80 \%$, range 53-100\%) than in patients without MS (mean $16 \%$, range $0-34 \%$ ).

In this study, periventricular location of the central vein sign was more obvious in MS lesions (55.6\%) than in CSVD lesions (40.5\%). This matches with previous studies done by Sparacia et al. [14], Lane et al. [16], Kau et al. [17], and Al-Zandi et al. [5]. This observation reflects the fact that MS inflammation begins around small cerebral veins, as discovered by pathology $[11,18]$.

This study also revealed that the CVS is a significant discriminator $(p<0.001)$ between MS-WMLs and CSVD-WMLs with a sensitivity $83.3 \%$, specificity $70 \%$, positive predictive value $80.6 \%$, and negative predictive value $73.7 \%$. The CVS was also found to have a premium diagnostic efficacy in MS diagnosis with a specificity reaching $100 \%$ when the load of CVS positive lesions is $\geq 44.24 \%$. This finding is in agreement with the results

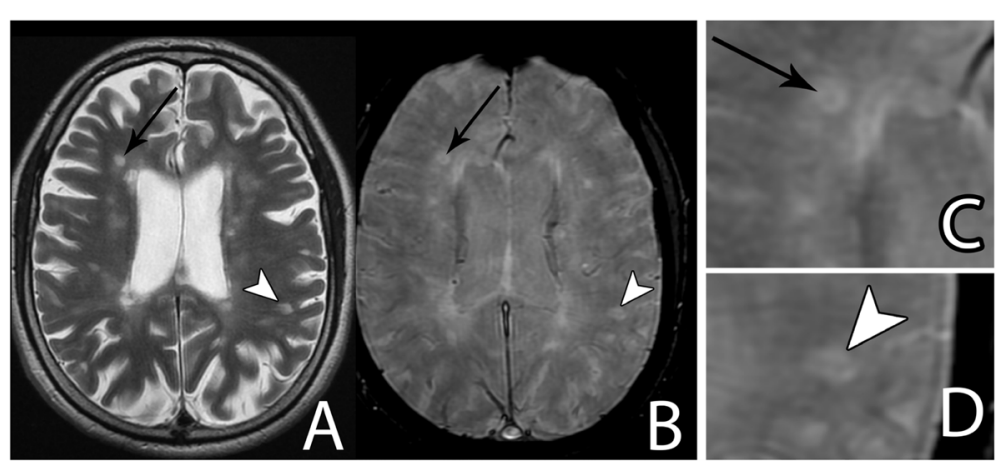

Fig. 5 Axial (a) T2WI image shows hyperintense MS periventricular and juxta-cortical lesions (arrow and arrowhead). On the corresponding SWI (b) and amplified SWI images (c and $\mathbf{d}$ ), a thin hypointense line and a central hypointense dot (representing the CVS) are seen in the juxtacortical (arrow head) and periventricular lesions (arrow), respectively 




Fig. 6 A 60-year-old male patient with cerebral small vessel disease. Axial (a and c) T2Wl images show multiple hyperintense periventricular (black arrows), deep white matter (arrow head), and juxta-cortical lesions (white arrow). On corresponding (b and d) SWI images, a thin hypointense line representing the CVS is seen in the periventricular (black arrows), deep white matter (arrow head), and juxta-cortical lesions (white arrow)

of Al-Zandi et al. study [5] in which the specificity for predicting the diagnosis of MS reached $100 \%$ when the load of CVS positive lesions was $\geq 47.5 \%$. Also the sensitivity, specificity, positive, and negative predictive values of the CVS in MS diagnosis were $86.84 \%, 86.6 \%, 88.7 \%$,

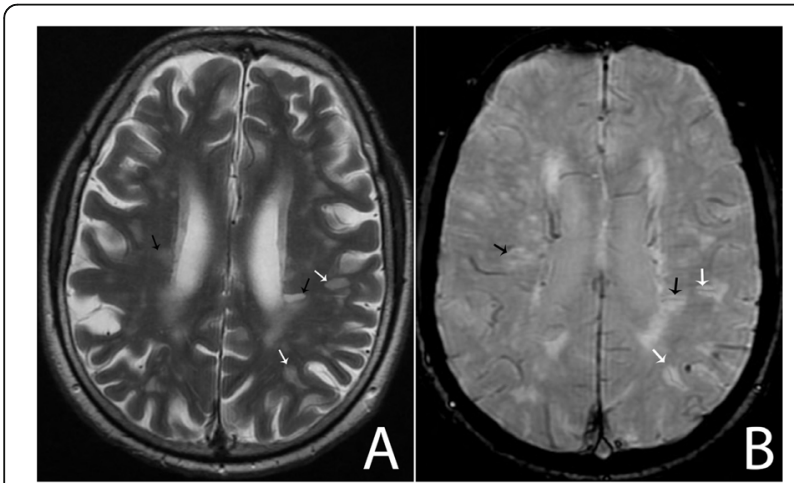

Fig. 7 A 63-year-old female patient with cerebral small vessel disease. Axial (a) T2WI image show multiple hyperintense periventricular (black arrows) and juxta-cortical lesions (white arrow). On corresponding (b) SWI image, a thin hypointense line representing the CVS is seen in the periventricular (black arrows) and juxta-cortical lesions (white arrow)
Table 4 The sensitivity, specificity, PPV, NPV and AUC for central vein sign in MS patients

\begin{tabular}{lllllll}
\hline & $\begin{array}{l}\text { AUC } \\
(95 \% \mathrm{Cl})\end{array}$ & $p$ value & Sensitivity & Specificity & PPV & NPV \\
& & & & & \\
$\begin{array}{l}\text { Percentage of } \\
\text { CVS SWI }\end{array}$ & $\begin{array}{l}0.863 \\
(0.76-0.96)\end{array}$ & $0.001^{*}$ & 83.3 & 70.0 & 80.673 .7 \\
\hline
\end{tabular}

and $75.72 \%$, respectively. Yet, with regard to the specificity of the CVS, our results did not cope with a study done by Lummel et al. [19] which revealed that the central vein sign is a non-specific finding for MS. A probable explication for this result is that they used a different technique (susceptibility-weighted imaging angiography), that differs from SWI applied in our study.

The limitations of this study can be summarized in three main factors: first, the relatively low field strength $(1.5 \mathrm{~T})$ compared to other studies done using field strength $3 \mathrm{~T}$ and $7 \mathrm{~T}$. Second, although we carried out a lesion based analysis in a reasonable number of WMLs, both MS and CSVD groups were small. Eventually, the significant difference in age between the two groups of patients that reflects the different age of affection in MS and CSVD.

\section{Conclusion}

Finally, it was concluded that SWI sequence improves the diagnostic accuracy of MS through detection of the CVS which considered as a fairly reliable discriminator between MS-WMLs and CSVD-WMLs especially when CVS positive lesion load is $\geq 44.24 \%$ of the total lesions.

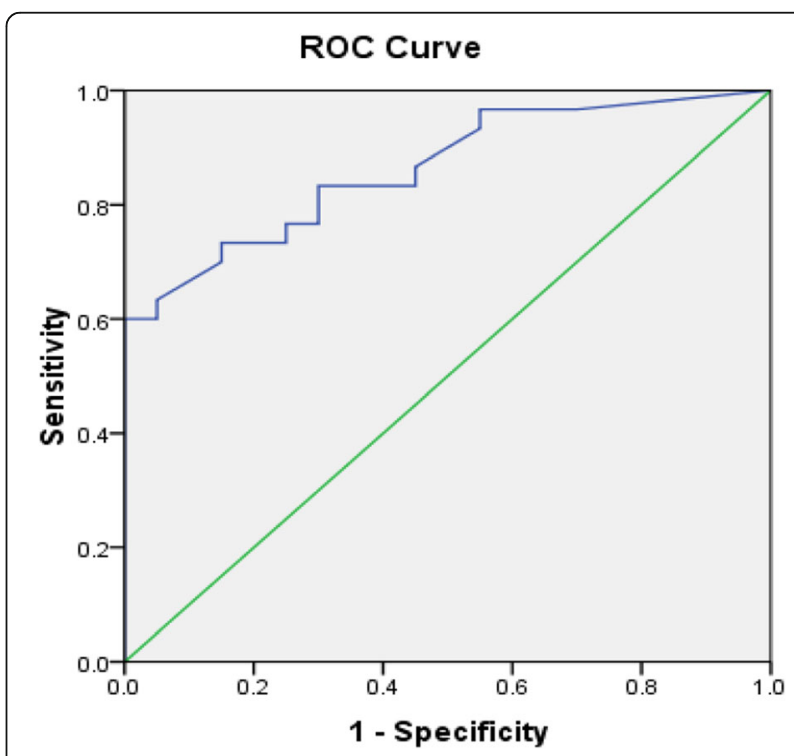

Fig. 8 ROC curve for determination of CVS sensitivity and specificity in MS diagnosis 


\section{Abbreviations}

CSVD: Cerebral small vessel disease; CVS: Central vein sign; MRI: Magnetic resonance imaging; MS: Multiple sclerosis; SWI: Susceptibility weighted imaging; WMLs: White matter lesions

\section{Acknowledgements}

Not applicable.

\section{Authors' contributions}

SE and RA designed the research. RA performed the research; and wrote the manuscript. SE and ME analyzed the collected data. SE, TB, and ME revised data and manuscript. All authors read and approved the final manuscript.

\section{Funding}

Not applicable (no funding received for this study).

\section{Availability of data and materials}

Available on request with the corresponding author.

\section{Ethics approval and consent to participate}

This study was approved by the research ethics committee of the Radiology Department of the Faculty of Medicine, Mansoura University, on 8/7/2017, reference number of approval: MD/17.06.94.

All patients included in this study gave a written informed consent to participate in the research.

\section{Consent for publication}

All patients included in this study gave a written informed consent to publish the data contained in this study.

\section{Competing interests}

The authors declare that they have no competing interests.

\section{Author details}

'Specialist of Diagnostic and Interventional Radiology, Ministry of Health, Cairo, Egypt. ${ }^{2}$ Diagnostic and Interventional Radiology, Mansoura University, Mansoura, Egypt. ${ }^{3}$ Neurology, Mansoura University, Mansoura University, Mansoura, Egypt.

Received: 20 January 2020 Accepted: 15 April 2020

Published online: 23 April 2020

\section{References}

1. Sheng H, Zhao B, Ge Y (2019) Blood perfusion and cellular microstructural changes associated with iron deposition in multiple sclerosis lesions. Frontiers in neurology, 10, 747. https://doi.org/10.3389/fneur.2019.00747.

2. Filippi M, Rocca MA, Ciccarelli O, De Stefano N, Evangelou N, Kappos L et al (2016) MRI criteria for the diagnosis of multiple sclerosis: MAGNIMS consensus guidelines. Lancet Neurology 15(3):292-303 https://doi.org/10. 1016/S1474-4422(15)00393-2

3. Miller DH (2004) Biomarkers and surrogate outcomes in neurodegenerative disease: lessons from multiple sclerosis. NeuroRx 1(2):284-294 https://doi org/10.1602/neurorx.1.2.284

4. Giovannoni G, Butzkueven H, Dhib-Jalbut S, Hobart J, Kobelt G, Pepper G et al (2016) Brain health: time matters in multiple sclerosis. Multiple sclerosis and related disorders 9:S5-S48 https://doi.org/10.1016/..msard.2016.07.003

5. Al-Zandi SH, Fayadh NA, Al-Waely NK (2018) Central vein sign detected by SWI at 3 T MRI as a discriminator between multiple sclerosis and leukoaraiosis. The Egyptian Journal of Radiology and Nuclear Medicine 49(1):158-164 https://doi.org/10.1016/.j.jrnm.2017.09.003

6. Absinta M, Sati P, Gaitán MI, Maggi P, Cortese IC, Filippi M et al (2013) Seven-tesla phase imaging of acute multiple sclerosis lesions: A new window into the inflammatory process. Annals of neurology 74(5):669-678 https://doi.org/10.1002/ana.23959

7. Sati P, Thomasson DM, Li N, Pham DL, Biassou NM, Reich DS et al (2014) Rapid, high-resolution, whole-brain, susceptibility-based MRI of multiple sclerosis. Multiple Sclerosis Journal 20(11):1464-1470 https://doi.org/10. $1177 / 1352458514525868$

8. Miller C, Goldberg MF (2012) Susceptibility-weighted imaging and computed tomography perfusion abnormalities in diagnosis of classic migraine. Emergency radiology 19(6):565-569. https://doi.org/10.1007/ s10140-012-1051-2 Epub 2012 Jun 9

9. Fazekas G, Fazekas F, Schmidt R, Kapeller P, Offenbacher H, Krejs GJ (1995) Brain MRl findings and cognitive impairment in patients undergoing chronic hemodialysis treatment. Journal of the neurological sciences 134(1): 83-88 https://doi.org/10.1016/0022-510X(95)00226-7

10. Forbes K (2017) MRI brain white matter change: spectrum of change-how can we grade? JOURNAL OF THE ROYAL COLLEGE OF PHYSICIANS OF EDINBURGH 47(3):271. https://doi.org/10.4997/JRCPE.2017.313

11. Sati P, Oh J, Constable RT, Evangelou N, Guttmann CR, Henry RG et al (2016) The central vein sign and its clinical evaluation for the diagnosis of multiple sclerosis: a consensus statement from the North American Imaging in Multiple Sclerosis Cooperative. Nature Reviews Neurology 12(12):714. https://doi.org/10.1038/nrneurol.2016.166 Epub 2016 Nov 11

12. Mistry N, Abdel-Fahim R, Samaraweera A, Mougin O, Tallantyre E, Tench C et al (2016) Imaging central veins in brain lesions with 3-T T2*-weighted magnetic resonance imaging differentiates multiple sclerosis from microangiopathic brain lesions. Multiple Sclerosis Journal 22(10):1289-1296 https://doi.org/10.1177/1352458515616700

13. Geraldes R, Ciccarelli O, Barkhof F, De Stefano N, Enzinger C, Filippi M et a (2018) The current role of MRI in differentiating multiple sclerosis from its imaging mimics. Nature Reviews Neurology 14(4):199 https://doi.org/10. 1038/nrneurol.2018.14

14. Sparacia G, Agnello F, Gambino A, Sciortino M, Midiri M (2018) Multiple sclerosis: High prevalence of the 'central vein'sign in white matter lesions on susceptibility-weighted images. The neuroradiology journal 31(4):356361 https://doi.org/10.1177/1971400918763577

15. Tallantyre EC, Dixon JE, Donaldson I, Owens T, Morgan PS, Morris PG et al (2011) Ultra-high-field imaging distinguishes MS lesions from asymptomatic white matter lesions. Neurology, 76(6), 534-539. https://doi.org/10.1212/ WNL.0b013e31820b7630

16. Lane Jl, Bolster B, Campeau NG, Welker KM, Gilbertson JR (2015) Characterization of multiple sclerosis plaques using susceptibility-weighted imaging at 1.5 T: can perivenular localization improve specificity of imaging criteria? Journal of computer assisted tomography 39(3):317-320. https:// doi.org/10.1097/RCT.0000000000000233

17. Kau T, Taschwer M, Deutschmann H, Schönfelder M, Weber JR, Hausegger KA (2013) The "central vein sign": is there a place for susceptibility weighted imaging in possible multiple sclerosis? European radiology 23(7):1956-1962. https://doi.org/10.1007/s00330-013-2791-4 Epub 2013 Feb 22

18. Adams CWM, Poston RN, Buk SJ (1989) Pathology, histochemistry and immunocytochemistry of lesions in acute multiple sclerosis. Journal of the neurological sciences 92(2-3):291-306 https://doi.org/10.1016/0022$510 \times(89) 90144-5$

19. Lummel N, Boeckh-Behrens T, Schoepf V, Burke M, Brückmann H, Linn J (2011) Presence of a central vein within white matter lesions on susceptibility weighted imaging: a specific finding for multiple sclerosis? Neuroradiology 53(5):311-317 https://doi.org/10.1007/s00234-010-0736-z

\section{Publisher's Note}

Springer Nature remains neutral with regard to jurisdictional claims in published maps and institutional affiliations.

\section{Submit your manuscript to a SpringerOpen ${ }^{\circ}$ journal and benefit from:}

- Convenient online submission

- Rigorous peer review

- Open access: articles freely available online

- High visibility within the field

- Retaining the copyright to your article

Submit your next manuscript at $\boldsymbol{\nabla}$ springeropen.com 